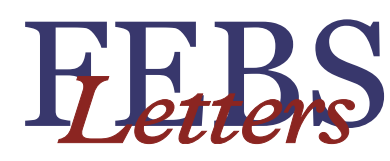

journal homepage: www.FEBSLetters.org

Review

\title{
Lipid droplets: A dynamic organelle moves into focus
}

\author{
Mathias Beller*, Katharina Thiel, Peter J. Thul, Herbert Jäckle \\ Max-Planck-Institut für biophysikalische Chemie, Abteilung für molekulare Entwicklungsbiologie, Am Fassberg 11, 37077 Göttingen, Germany
}

\section{A R T I C L E I N F O}

\section{Article history:}

Received 16 February 2010

Revised 15 March 2010

Accepted 16 March 2010

Available online 18 March 2010

Edited by Wilhelm Just

\section{Keywords:}

Lipid droplet

Organelle interaction

Endoplasmic reticulum

Vesicles

\begin{abstract}
A B S T R A C T
Lipid droplets (LDs) were perceived as static storage deposits, which passively participate in the energy homeostasis of both cells and entire organisms. However, this view has changed recently after the realization of a complex and highly dynamic LD proteome. The proteome contains key components of the fat mobilization system and proteins that suggest LD interactions with a variety of cell organelles, including the endoplasmic reticulum, mitochondria and peroxisomes. The study of LD cell biology, including cross-talk with other organelles, the trafficking of LDs in the cell and regulatory events involving the $L D$ coat proteins is now on the verge of leaving its infancy and unfolds that LDs are highly dynamic cellular organelles.
\end{abstract}

๑) 2010 Federation of European Biochemical Societies. Published by Elsevier B.V. All rights reserved.

\section{Lipid droplets}

Lipid droplets (LDs) are the lipid storage organelles of all organisms (reviewed in: [1]). They are important for the survival of the organism when food supplies are limited or energy consumption suddenly spikes. Stored lipids also serve to fuel developmental processes both in egg-laying animals and in plants. In addition, LDs play a protective role by scavenging free fatty acids, which otherwise are driven into non-oxidative pathways to form reactive lipids that promote cellular dysfunction (lipotoxicity) and eventually cell death (lipoapoptosis) [2].

Thus, while lipid storage is an elementary feature of life, it must be tightly controlled. Excessive storage of lipids, for example, can be detrimental to the organism, a phenomenon that is most obvious in emerging metabolic diseases such as atherosclerosis, diabetes and obesity. At the other end of the spectrum, the inability to store lipids in diseases such as lipodystrophy also results in multifaceted systemic problems [3]. The apparent need to tightly regulate energy balance in organisms obviously demands a finely tuned regulation of lipid storage and mobilization which ultimately translates into processes such as LD biogenesis and LD filling with fat on the one hand, and fat mobilization from LDs and their breakdown on the other.

Although all cell types are probably able to store fat in LDs, at least temporarily, these organelles are usually highly enriched in specialized cell types such as the mammalian adipocyte or corresponding fat body cells in insects. Despite variations in size (from

\footnotetext{
* Corresponding author. Fax: +49551 2011755.

E-mail address: mbeller@gwdg.de (M. Beller).
}

the sub- $\mu \mathrm{m}$ to $200 \mu \mathrm{m}$ in diameter) and appearance in virtually every organism and cell type, the structure and organization of LDs is highly conserved. They consist of a hydrophobic core of stored fat, mostly neutral lipids such as triacylglycerols but also sterols and sterol esters, which is surrounded by a protein-bearing phospholipid hemimembrane (reviewed in: [4]). Among all known cytoplasmic organelles, LDs are exceptional in that they possess only a monolayer membrane.

How are these organelles formed? The most widely accepted model of LD biogenesis involves the incorporation of neutral lipids in the interspace between the bilayer leaflets of the ER membrane, followed by a budding-out of the cytoplasm-oriented hemimembrane to form the fat-bearing LD (Fig. 1(1)). In this model, the cytoplasmic leaflet of the ER membrane provides the phospholipid monolayer surface of the nascent $\mathrm{LD}$. The origin of the protein coat of LDs is not yet fully understood. It seems likely, however, that the coating process involves two different pathways. Some LD coat proteins were recently shown to be inserted into the ER membrane before localization to the LD occurs (Fig. 11) [5,6], whereas other proteins, such as the PAT-domain protein perilipin/PLIN1, are never found in association with the ER [7]. The details of how these proteins attach to or insert into the LD membrane have not yet been explored in any detail. Once formed and released from ER, cytoplasmic LDs are likely to increase their volume either by localized lipid synthesis [8] or by fusion of LDs [9] (Fig. 12). Upon cellular demand, i.e. when energy is needed by the cell, stored lipids are remobilized by the tightly controlled activity of specific lipases. In the mammalian adipocyte in particular, both the executing enzymes and the regulatory pathways have been intensively studied and have already been the subject of reviews (see [10]). 


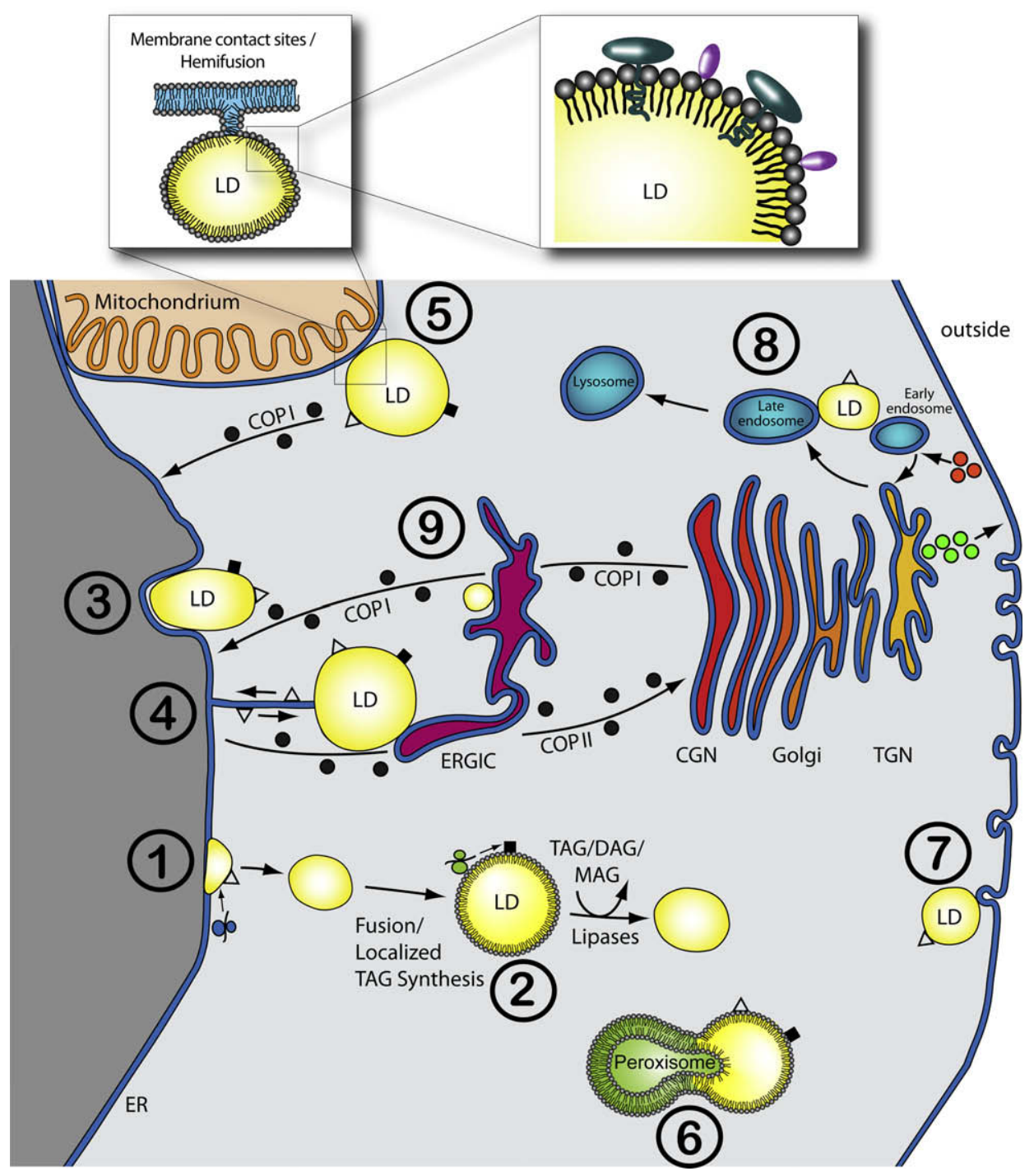

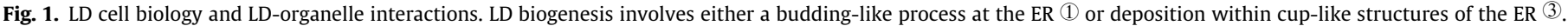

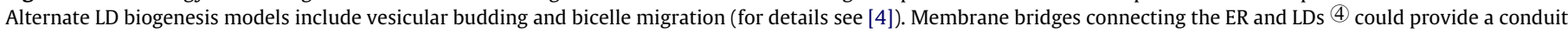

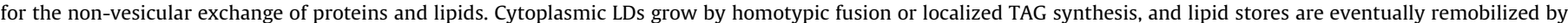

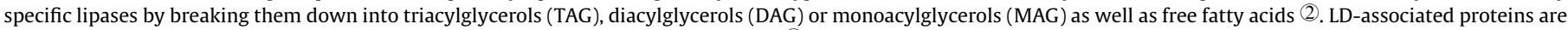

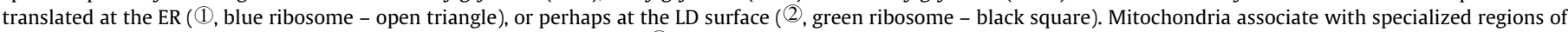

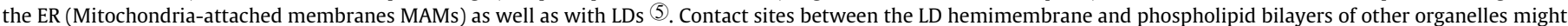

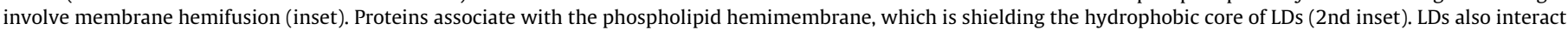

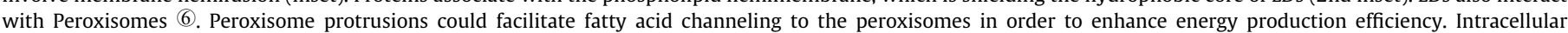

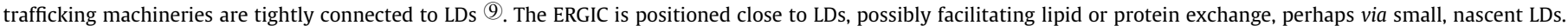

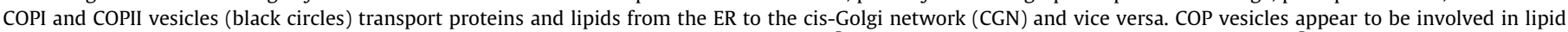

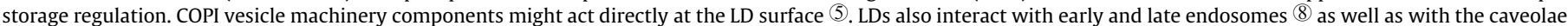

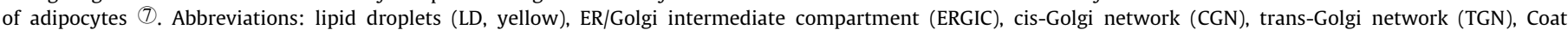

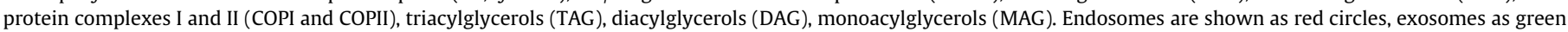
circles.

Additionally, an important contribution of autophagy to regulated LD remobilization was recently demonstrated [11]. The fatty acids liberated by LD breakdown are subsequently utilized by the cell as an energy source and/or for metabolite production, or they are shuttled outside the cell to be used in other cells or target tissues.

While the LD life cycle as a whole seems to be clear, many steps in the various processes are still unknown or controversial. For example, it has also been suggested that LDs are formed in socalled ER cups without undergoing a budding-out process [12] (Fig. 1(3). Also vesicular budding and bicelle formation at the ER membrane were discussed as models for LD biogenesis [4]. In addition, mechanistic details of the cellular control of LD size and num- bers are largely unknown, as are the mechanism(s) and signaling pathways that regulate the LD protein coating. Furthermore, only vaguely defined sequence characteristics of coat proteins have been identified so far. These include hydrophobic or basic amino acid stretches that can reside in various positions of a given $L D$ coat protein (e.g. [5,13-16]), raising the question of how LD-specific targeting can be achieved in the absence of a defined LD localization sequence. The answer to this question seems to be rather complex, since several of the proteins that have been identified are found in association with only a subset of the cellular LDs $[17,18]$. It is therefore possible that the LDs within a cell differ distinctly with respect to function, maturation and/or metabolic status, and that 
these characteristics are reflected in LD-specific protein coat compositions. Identification and functional characterization of distinct marker proteins for subsets of LDs are now necessary in order to test whether there are indeed functionally distinct subclasses of LDs. In view of the rapidly increasing evidence for a complex functional repertoire of LDs, different LD subclasses would not come as a surprise, and they would continue to change the scope of LD biology much as perilipin/PLIN1 did when it was shown to act as a gatekeeper of mammalian lipolysis regulation $[19,20]$.

\section{LD coat proteins open a window on subcellular interactions of LDs}

Almost two decades ago, Londos and co-workers stimulated interest in the regulatory potential associated with the LD protein coat by identifying perilipin/PLIN1 as a major phosphoprotein of mammalian adipocytes [21]. Perilipin/PLIN1 serves as a gatekeeper and regulatory scaffold for basal and stimulated lipolysis regulation (reviewed in: [22]). Most studies on LD-associated proteins subsequently focused on perilipin/PLIN1 function to reveal its key role in the regulation of lipolysis in adipocytes. These studies, in turn, led to a growing interest in LD-associated proteins and the discovery of a new and evolutionarily conserved perilipin-like protein family [22]. These proteins have a common but functionally not yet fully characterized N-terminal domain of about 100 amino acids, the PAT-domain, which is named after the three main mammalian family representatives perilipin/PLIN1, ADRP/Adipophilin/PLIN2 and Tail interacting protein of $47 \mathrm{kDa}$ (Tip47/PLIN3). PAT-domain proteins are capable of associating with LDs and participate in the regulation of cellular lipid metabolism in organisms as evolutionarily distant as insects [23,24] and mammals (reviewed in: [22]).

In an attempt to identify additional components of the LD protein coat that participate in the regulation of fat-dependent cellular and organismic energy homeostasis, i.e. the control of fat storage and fat mobilization, several laboratories have characterized LDassociated proteins from various organisms. LD fractions were purified by sucrose-gradient ultracentrifugation and analyzed by mass spectrometry (e.g. [17,25-29]). Among the several hundred proteins identified by these studies, a relatively small core set of LD-associated proteins was consistently found. It includes the abovementioned PAT-domain proteins, certain lipases and other metabolic enzymes. In addition, a variety of proteins specific to the cell type and the approach taken were observed, including reproducible sets of proteins which appeared at a first glance to be contaminants. These included proteins of ribosomal and mitochondrial origin, ER-localized components and traffickingassociated proteins [17,25-29]. Finally, some totally unexpected proteins were found as well, such as histones [27], suggesting that the majority of the identified proteins may derive from contaminations of LDs. These contaminations might have been the result of the extreme hydrophobic properties of LDs which allow them to associate with certain types of proteins and/or organelle remnants during the purification procedure. However, the demonstration that the most unexpected association, that between LDs and histones, is of functional relevance [27] requires a revisiting of the other previously suspected "contaminants" as well. In fact, there is now growing evidence suggesting that what were once presumed to be "contaminants" of the LD coat proteomes are in fact early indicators of functional interactions between LDs and various other organelles of the cell.

\section{Lipid droplets and Endoplasmic Reticulum: the elusive liaison}

The close apposition of LDs and the ER was first observed decades ago by both electron and light microscopy (e.g. [30]).
LD proteomes also showed a significant enrichment of proteins predicted or already known to localize at the ER [29]. A tight connection between ER and LDs, as suggested by both the visual and biochemical studies, is now supported by several functional studies. Genomic screens designed to isolate possible key regulators of LD morphogenesis identified, for example, the Fldp1/Seipin protein in yeast [31,32]. Loss-of-function mutations of Fldp1/Seipin cause irregularly shaped, giant LDs, which are often clustered alongside proliferated ER [31,32]. In humans, mutant Fldp1/Seipin protein was found in patients suffering from the most severe form of hereditary lipodystrophy, Berardinelli-Seip Congenital Lipodystrophy type 2 (BSCL2) [33]. The virtual absence of adipose tissue seen in such patients has been associated with a proposed role of Seipin in the differentiation of mesenchymal cells into preadipocytes [34]. In fibroblasts of BSCL2 patients, numerous small, irregularly shaped LDs are apparent [32]. In yeast, Seipin is localized in the ER. It forms puncta in positions where LDs adhere, suggesting a function in LD maintenance, assembly and/or shape determination. Moreover, Seipin affects the phospholipid and triglyceride composition of LDs, which in turn could play an important role in preventing their fusion $[31,35]$. In fact, LD fusion is a hallmark of Seipin mutants and is likely to be the cause of the giant LDs that were observed [31].

A guiding role of the ER in LD biogenesis and the initial protein coating of the LD surface can also be deduced from recent studies on proteins that were identified by LD proteomics $[6,13]$. These studies not only uncovered hydrophobic and basic amino acid stretches from Caveolin, UBXD8 and AAM-B, which were shown to be necessary and sufficient for LD localization, but also tested the proposed LD-protein localization mechanism via a transient insertion of UBXD8 and AAM-B in the ER membrane. Most importantly, the authors characterized not only this localization path of the proteins but also performed experiments whose results allow the postulation of a bidirectional trafficking of proteins to and from the LDs in response to the nutritional status of the cells [6]. Such a transport might involve an intimate functional connection between LDs and ER by membrane bridges or at least through a very close apposition that allows a direct contact among the partners (Fig. 14) [6].

The characterization of such contact sites might shed light on the question of whether and how LDs arise at specific sites of the ER, as suggested by the localization of Seipin. Such sites of LD biogenesis might differ in their phospholipid composition, defining specific sites, such as "rafts" (reviewed in: [36]), for the assembly of a LD biogenesis machinery. A spatial restriction of the LD biogenesis machinery within the ER is in line with the current model of how LDs are formed, since the accumulating neutral lipid in the ER intermembrane space would otherwise eventually spread further in the hydrophobic environment instead of nucleating the proposed LD "lens". As a result, the incoming lipids would expand and thereby spread the ER hemimembranes, consistent with a phenotype as observed in the Seipin mutants.

Lipidomics should help to uncover LD biogenesis sites. In fact, previous lipidomics studies demonstrated a complex lipid composition of LDs $[37,38]$ and suggested specific sites of the ER as the birthplace of LDs, since the phospholipid composition of LDs was markedly different to the composition of ER membranes [38]. It remains to be elucidated whether the different lipid compositions reflect only the heterogeneity of the ER, i.e. differences between rough and smooth ER, or depend on sites where LDs are generated. Recently developed microanalytical tools for lipid analysis [39] will allow a more comprehensive study of the lipid composition of LDs from different cell types and organs to close this important gap in our knowledge and to finally characterize the proposed birth place of the LDs. 


\section{Interactions between LDs and other organelles - the unfolding story}

Interactions between LDs and organelles other than the ER are less well documented and rely almost completely on microscopy and proteomics studies rather than functional data. However, an interaction between LDs and the mitochondria would be functionally reasonable, since a flow of freed fatty acids derived from LD "breakdown" could be directly channeled into energy production via beta-oxidation. In fact, at least two lines of evidence provide additional support for a tight connection between mitochondria and LDs. First, LDs are transported via microtubule tracks towards the mitochondria in steroidogenic cells, where the fatty acids from LDs are channeled into the synthesis of steroid hormones [40]. Furthermore, a lipogenic enzyme called diacylglycerol acyl transferase 2 is found in mitochondria-associated membranes (MAMs) of the ER, which are specialized regions for lipid biosynthesis (Fig. 15). DGAT2 was found to translocate from the MAMs to LDs when LD biogenesis is stimulated upon oleic acid treatment of cells $[8,41]$.

A link between LD breakdown and energy production was also described in the yeast Saccharomyces cerevisiae for LDs and peroxisomes. As observed with mitochondria, a close apposition between LDs and peroxisomes was noted [42]. Most interestingly, electron microscopy revealed peroxisome protrusions, so-called pexopodia, which extend into the LD core (Fig. 16) [42]. A failure in peroxisome function had severe consequences for LD morphology, leading to elongated, curled and tangled electron-dense regions within the LD core, which were called "gnarls" by the authors [42]. Up to now it is not clear if similar interactions also exist in organisms other than yeast. However, it is worth noting that peroxisomes are thought to arise at the ER, implying that cell organelles specialized in lipid metabolism have a common cellular origin. This would allow for the coordinated biogenesis of the two organelles in response to regulatory inputs that depend on the energy state of a given cell.

The peculiar finding that ribosomal constituents are both enriched in the "hit lists" of functional genomic screens focusing on lipid storage phenotypes $[43,44]$ and in lipid droplet proteomics screens $[17,27,45]$ raises the possibility of a direct and functional association between ribosomes and LDs. The abovementioned findings are supported by electron microscopy and hybridization techniques showing that ribosomes as well as RNA molecules are found in contact with LDs in mast cells [46] and leukocytes [45]. Also, in cells infected with Hepatitis $C$ virus, the RNA of the virus was detected at specific ER sites, often juxtaposed to LDs [47]. It is therefore reasonable to speculate that at least some of the components of the LD protein coat are targeted by localized translation (Fig. 1(2).

\section{Docking of LDs to other organelles}

How is the exchange of proteins and lipids between LDs and the interacting organelles achieved? One possible mechanism could involve so-called "membrane contact sites" (MCS), as initially proposed by Levine and co-workers (reviewed in: [48]). Such sites have been described for different organelles such as ER and mitochondria forming the MAMs described above, which facilitate the non-vesicular transfer of phosphatidylserine [48]. Interactions between LDs and bilayer-surrounded partner organelles might involve "hemifusion" (Fig. 15); inset Fig. 1), a mechanistic process proposed by Murphy and co-workers [49]. This process would involve a transient but continuous fusion of the phospholipid monolayer of LDs and the outer membrane leaflet of a docking vesicle/ organelle without any mixing of the contents of the interaction partners. However, in this process, proteins and possibly lipid frac- tions of the LD surface and corresponding components of the outer leaflet would be able to translocate from one interaction partner to the other. The transfer of Caveolin1 from caveolae to the unilocular LD in mammalian adipocytes has been suggested to invoke such a mechanism, although a vesicle-mediated transfer was also discussed (Fig. 17) [49]. Factors that mediate contact between LDs and their partners appear to be similar to or identical with those known to mediate vesicle docking and fusion (reviewed in: [50]). This proposal is consistent with the recent finding that the vesicle trafficking machinery appears to be important for $L D$ homeostasis (see below).

\section{Lipids on the move - LD transport and vesicle trafficking}

LDs travel within cells via cytoskeleton tracks such as the microtubules in diverse cellular and organismic systems such as fungi, Drosophila embryos and ovaries, fish eggs and cultured mammalian cells or the lactating mammary gland (reviewed in: [51]). Thus, LDs might be shuttled to cellular sites where stored lipids are remobilized to be further processed as shown in steroidogenic cells [40].

Movement of LDs also appears to be essential for LD biogenesis, since microinjection of anti-Dynein antibodies blocks LD formation/volume growth [50,51]. Growth of LDs is likely to involve homotypic fusion between pre-existing LDs, a process mediated by the SNAP23 protein [9]. The involvement of this protein, together with various vesicle transport-associated proteins such as other SNARE proteins and various Rabs found in LD proteomes, supports the possibility that the molecular machines that mediate vesicle trafficking, docking and fusion could also be active at the LD surface $[17,26,28,50,52]$. The presence of such proteins at the LD coat furthermore suggests that vesicle-associated proteins might mediate not only interactions between LDs, but also interactions between LDs and trafficking vesicles. In fact, a Rab-dependent interaction of early endosomes and LDs was demonstrated in a reconstituted in vitro system (Fig. 18) [52]. While transport vesicles do not seem to fuse with the LDs, an interaction between the two cellular components could serve as a means to exchange lipids and/or proteins, as outlined above. Such a lipid transport function was proposed for a novel class of Dpm1-positive transport vesicles in S. cerevisiae which mediate lipid transport to LDs [53].

Information about the pathways regulating LD-vesicle interactions is very limited. The small GTP binding protein ARFRP1 was only recently shown to be a regulator of $L D$ fusion, probably by acting upstream of SNAP23 [54]. Loss of Arfrp1 results in smaller LDs, a phenomenon probably based on fewer LD fusion events caused by, or linked to, the translocation of SNAP23 from LDs to the cytosol. In physiological terms, lack of Arfrp1 activity causes an increased basal lipolysis rate. The mechanism of ARFRP1 action is still unknown, but the authors speculate that ARFRP1 alters the attachment properties of Rab proteins to LDs [54]. Interestingly, ARFRP1 seems to function independently of COPI-dependent trafficking which was recently shown to participate in LD homeostasis $[43,44,55]$.

\section{COPI-dependent regulation of LD physiology}

Early electron microscopy studies of cells led to a vesicle transport hypothesis for protein trafficking involving three major classes of coated vesicles that had been purified: COPI- and COPIIcoated vesicles (where COP stands for coat protein complex) and clathrin-coated vesicles. In each type of vesicles, coat components are needed for the generation of highly curved membrane areas, recruitment of cargo (and the exclusion of non-cargo proteins/lipids), vesicle scission and uncoating factor recruitment. Transport of 
proteins and lipids between ER and the Golgi apparatus is provided by COPI and COPII vesicles (Fig. 19).

Systematic genome-wide RNA interference knock-down experiments with Drosophila cell lines revealed that components of the COPII vesicles that mediate the anterograde transport from ER to Golgi did not consistently affect the LD protein coat and LD function $[43,44]$. In contrast, COPI vesicles, which mediate the retrograde transfer from the Golgi to the ER (reviewed in: [56,57]), are essential for LD function $[43,44]$. Further experiments in mammalian cell lines showed evolutionary conservation of this function $[43,55]$.

COPI vesicles consist of a heptameric protein coat, the coatomer, and involve an assembly process at the Golgi, which is initiated by the activity of the small GDP/GTP binding protein Arf1. The Arf1 nucleotide binding state is regulated by a specific Arf GTPase activating enzyme (ARF GAP) and an Arf GDP/GTP exchange factor (ARF GEF) which control the bending and budding-off of the preassembled COPI vesicles from the Golgi [56,57]. Knock-down experiments by RNA interference of the COPI components caused (with the exception of $\varepsilon \mathrm{COP}$ ) increased lipid stores not only in Drosophila $[43,44]$ but also in different mammalian tissue culture cell lines $[43,55]$. Subsequent functional analyses revealed that the increased lipid storage is due to a decreased lipolytic rate in the cells which correlates with the inability of the Adipocyte Triglyceride Lipase (ATGL) to join the LD surface $[43,55]$. Indeed, COPI seems to play a more general role in proper protein assembly at the LD surface, since PAT proteins are also mislocalized in response to COPI perturbation [43,55]. Interestingly, however, COPI-dependent PAT-domain protein localization is cell-type specific, a phenomenon likely to reflect differing PAT-domain protein configurations and/or cell-specific differences in PAT-domain protein expression levels. In HeLa cells, for example, Tip47/PLIN3 localization at the LD surface is unaffected by COPI perturbation, whereas ADRP/ PLIN2 is no longer targeted to the LD [55]. HeLa cells express only small amounts of both ADRP/PLIN2 and Tip47/PLIN3. In mouse AML12 hepatocytes, however, ADRP/PLIN2 localization is not significantly affected, whereas the recruitment of Tip47/PLIN3 to the LD surface is strongly enhanced or its attachment is stabilized in response to reduced COPI activity [43]. AML12 cells normally contain high amounts of ADRP/PLIN2 and low amounts of Tip47/ PLIN3 attached to LDs and are marked by a high efficiency to accumulate LDs (C. Sztalryd, personal communication). We attribute the observed changes in the LD protein coat in response to altered COPI activity to a different and specific need for COPI for the localization of the different PAT proteins at the LD surface.

The current results showing effects of reduced or lacking COPI activity on LD function are not yet conclusive with respect to a possible direct role of COPI components in LD function. The effects could still be indirect and a consequence of the loss of canonical COPI function in retrograde Golgi/ER trafficking. However, two lines of evidence point towards a direct role of COPI at the LD surface. First, Arf1 was shown to localize to LDs in a nucleotide-dependent manner, and its absence affects ADRP localization [58]. Secondly, components of the COPI machinery were found in highly purified lipid droplet proteomes [26]. These findings are consistent with the argument that COPI acts directly at the LD surface, perhaps by mediating the transport of lipids or proteins to the COPI target destination, the ER. However, COPI-mediated transport from ER exit sites ("ERES") or from the ER-Golgi intermediate compartment ("ERGIC") to LDs still cannot be ruled out in view of a recent study by Soni et al. [55]. Their immunofluorescence and electron tomography studies revealed a close apposition of LDs with both ERES and the ERGIC. Thus, besides the possibility of COPI vesicle trafficking to or from the LDs, those findings are also consistent with a model proposing that small, nascent LDs formed at ERES or the ERGIC sites could contribute to larger, pre-existing LDs by
LD fusion. Future experiments will be needed to address these and other possible models that explain the results obtained with the manifold experimental approaches taken. Such studies should be open to the possibility that the COPI vesicle machinery may also participate in very different processes and trafficking routes, including trafficking from peroxisomes to the ER or peroxisome proliferation, as suggested in previous reports [59].

\section{Summary and perspectives}

LD research gained exciting perspectives. Although LDs were long regarded as static fat stores, recent studies clearly emphasize a highly dynamic role for these organelles in cellular energy homeostasis and even envision an important role of droplet-derived lipid modifications in cellular regulatory processes such as the transport of morphogen signaling ligands [60]. The unique feature of these organelles is their surface: a monolayer sheet of phospholipids probably originating from the cytoplasm-oriented leaflet of the ER. The available evidence is consistent with a mechanism that involves site-specific filling of the ER bilayer membrane interspace, bending of the outer leaflet and budding of lipid-filled vesicles surrounded by the ER-derived monolayer leaflet that may maintain a specific lipid and protein coat signature. Subsequent processes regulating the proper size and growth of the newly formed LDs are still unclear. However, increasing evidence suggests that such processes are under tight control, since whole-genome genetic screens $[31,32,43,44]$ have identified factors that affect those properties. Both genetic and LD proteome studies have revealed a number of factors that not only affect the size and shape of LDs but also the physiology of cells and energy homeostasis beyond the level of single cells. One prominent example is Brummer/ ATGL, an evolutionarily conserved lipase decorating the LD surfaces. In its absence, single cells accumulate excess fat and the entire organism develops an obese phenotype [61-63]. Conversely, overexpression of Brummer/ATGL reduces the fat content in single cells as well as the whole organism. This finding, obtained with a distinct and confirmed component of the LD protein coat, suggests that LDs are indeed sites of regulatory events and that they could contain entire molecular machines for regulating the energy homeostasis of cells and, together with the specialized adipocytes of the fat storage organ, of the entire organism.

The dynamics of LDs includes not only fat storage and mobilization control, but also an apparent direct and possibly functional interaction with other cell organelles, including peroxisomes and mitochondria in addition to ER. There is visual documentation that this interaction is quite intimate in the case of the peroxisomes and that it involves membrane attachment receptor proteins such as SNAP23 in the case of the mitochondria [64]. Needless to say, each and every one of these interactions could bear new surprises in terms of LD functions and regulatory pathways.

While this emerging picture of LDs is exciting, the current mosaic of information remains patchwork for the most part. Just as numerous steps in the LD life cycle still await elucidation, more work will be needed to determine whether LD trafficking and emerging LD-organelle interactions are indeed of functional significance and what function they may serve. Novel tools for visualizing LD dynamics such as specific dyes $[8,65]$ or recent enhancements of imaging techniques including coherent antistokes Raman scattering (CARS) microscopy (e.g. [66,67]) or electron tomography (e.g. $[55,68])$ might accelerate the deciphering of functional and mechanistic details.

The apparent simplicity of lipid droplets is eventually a tribute to their probable long evolutionary history as storing excessive nutrients must have been a very basic and early prerequisite of survival. We only started to appreciate the delicate regulatory sys- 
tem acting on those simple organelles to adjust the cellular and organismic lipid stores and only scratched the various LD functions, which developed on top of the energy storage function. Unraveling the basic principles underlying the past and current features of the cellular lipid store and its regulation will ultimately also result in a deeper understanding of pathophysiological conditions including obesity and ectopic fat deposition with all of their detrimental consequences such as atherosclerosis, insulin resistance or diabetes. A better understanding of the cell biology of LDs may thus be the key to developing novel therapies addressing these pressing health issues.

\section{Acknowledgements}

This work was supported by the Max Planck Society. The authors would like to thank $\mathrm{H}$. Sebesse for help with the illustration and C. Sztalryd for critical reading of the manuscript.

We apologize to the colleagues whose research articles could not be cited due to space constraints.

\section{References}

[1] Murphy, D.J. (2001) The biogenesis and functions of lipid bodies in animals, plants and microorganisms. Prog. Lipid Res. 40, 325-438.

[2] Kusminski, C.M., Shetty, S., Orci, L., Unger, R.H. and Scherer, P.E. (2009) Diabetes and apoptosis: lipotoxicity. Apoptosis 14, 1484-1495.

[3] Herranz, P., de Lucas, R., Perez-Espana, L. and Mayor, M. (2008) Lipodystrophy syndromes. Dermatol. Clin. 26, 569-578. ix.

[4] Walther, T.C. and Farese Jr., R.V. (2009) The life of lipid droplets. Biochim. Biophys. Acta 1791, 459-466.

[5] Horiguchi, Y., Araki, M. and Motojima, K. (2008) Identification and characterization of the ER/lipid droplet-targeting sequence in 17betahydroxysteroid dehydrogenase type 11 . Arch. Biochem. Biophys. 479, 121-130.

[6] Zehmer, J.K., Bartz, R., Bisel, B., Liu, P., Seemann, J. and Anderson, R.G. (2009) Targeting sequences of UBXD8 and AAM-B reveal that the ER has a direct role in the emergence and regression of lipid droplets. J. Cell Sci. 122, 3694-3702.

[7] Londos, C., Brasaemle, D.L., Schultz, C.J., Segrest, J.P. and Kimmel, A.R. (1999) Perilipins, ADRP, and other proteins that associate with intracellular neutral lipid droplets in animal cells. Semin. Cell Dev. Biol. 10, 51-58.

[8] Kuerschner, L., Moessinger, C. and Thiele, C. (2008) Imaging of lipid biosynthesis: how a neutral lipid enters lipid droplets. Traffic 9, 338-352.

[9] Boström, P. et al. (2007) SNARE proteins mediate fusion between cytosolic lipid droplets and are implicated in insulin sensitivity. Nat. Cell Biol. 9, 12861293.

[10] Bezaire, V. and Langin, D. (2009) Regulation of adipose tissue lipolysis revisited. Proc. Nutr. Soc. 68, 350-360.

[11] Singh, R. et al. (2009) Autophagy regulates lipid metabolism. Nature 458, 1131-1135.

[12] Robenek, H., Hofnagel, O., Buers, I., Robenek, M.J., Troyer, D. and Severs, N.J. (2006) Adipophilin-enriched domains in the ER membrane are sites of lipid droplet biogenesis. J. Cell Sci. 119, 4215-4224.

[13] Ingelmo-Torres, M. et al. (2009) Hydrophobic and basic domains target proteins to lipid droplets. Traffic 10, 1785-1801.

[14] Zehmer, J.K., Bartz, R., Liu, P. and Anderson, R.G. (2008) Identification of a novel N-terminal hydrophobic sequence that targets proteins to lipid droplets. J. Cell Sci. 121, 1852-1860.

[15] Subramanian, V., Garcia, A., Sekowski, A. and Brasaemle, D.L. (2004) Hydrophobic sequences target and anchor perilipin A to lipid droplets. J. Lipid Res. 45, 1983-1991.

[16] Nakamura, N. and Fujimoto, T. (2003) Adipose differentiation-related protein has two independent domains for targeting to lipid droplets. Biochem. Biophys. Res. Commun. 306, 333-338.

[17] Beller, M., Riedel, D., Jänsch, L., Dieterich, G., Wehland, J., Jäckle, H. and Kühnlein, R.P. (2006) Characterization of the Drosophila lipid droplet subproteome. Mol. Cell. Proteomics 5, 1082-1094.

[18] Wolins, N.E., Brasaemle, D.L. and Bickel, P.E. (2006) A proposed model of fat packaging by exchangeable lipid droplet proteins. FEBS Lett. 580, 5484-5491.

[19] Tansey, J.T. et al. (2001) Perilipin ablation results in a lean mouse with aberrant adipocyte lipolysis, enhanced leptin production, and resistance to diet-induced obesity. Proc. Natl. Acad. Sci. USA 98, 6494-6499.

[20] Martinez-Botas, J. et al. (2000) Absence of perilipin results in leanness and reverses obesity in Lepr(db/db) mice. Nat. Genet. 26, 474-479.

[21] Greenberg, A.S., Egan, J.J., Wek, S.A., Garty, N.B., Blanchette-Mackie, E.J. and Londos, C. (1991) Perilipin, a major hormonally regulated adipocyte-specific phosphoprotein associated with the periphery of lipid storage droplets. J. Biol. Chem. 266, 11341-11346.

[22] Bickel, P.E., Tansey, J.T. and Welte, M.A. (2009) PAT proteins, an ancient family of lipid droplet proteins that regulate cellular lipid stores. Biochim. Biophys. Acta 1791, 419-440.
[23] Grönke, S., Beller, M., Fellert, S., Ramakrishnan, H., Jäckle, H. and Kühnlein, R.P. (2003) Control of fat storage by a Drosophila PAT domain protein. Curr. Biol. $13,603-606$

[24] Teixeira, L., Rabouille, C., Rorth, P., Ephrussi, A. and Vanzo, N.F. (2003) Drosophila Perilipin/ADRP homologue Lsd2 regulates lipid metabolism. Mech. Dev. 120, 1071-1081.

[25] Hodges, B.D. and Wu, C.C. (2010) Proteomic insights into an expanded cellular role for cytoplasmic lipid droplets. J. Lipid Res. 51, 262-273.

[26] Bartz, R., Zehmer, J.K., Zhu, M., Chen, Y., Serrero, G., Zhao, Y. and Liu, P. (2007) Dynamic activity of lipid droplets: protein phosphorylation and GTP-mediated protein translocation. J. Proteome Res. 6, 3256-3265.

[27] Cermelli, S., Guo, Y., Gross, S.P. and Welte, M.A. (2006) The lipid-droplet proteome reveals that droplets are a protein-storage depot. Curr. Biol. 16, 1783-1795.

[28] Brasaemle, D.L., Dolios, G., Shapiro, L. and Wang, R. (2004) Proteomic analysis of proteins associated with lipid droplets of basal and lipolytically stimulated 3T3-L1 adipocytes. J. Biol. Chem. 279, 46835-46842.

[29] Wu, C.C., Howell, K.E., Neville, M.C., Yates 3rd, J.R. and McManaman, J.L. (2000) Proteomics reveal a link between the endoplasmic reticulum and lipid secretory mechanisms in mammary epithelial cells. Electrophoresis 21, 3470-3482.

[30] Cushman, S.W. (1970) Structure-function relationships in the adipose cell. I. Ultrastructure of the isolated adipose cell. J. Cell Biol. 46, 326-341.

[31] Fei, W. et al. (2008) Fld1p, a functional homologue of human seipin, regulates the size of lipid droplets in yeast. J. Cell Biol. 180, 473-482.

[32] Szymanski, K.M. et al. (2007) The lipodystrophy protein seipin is found at endoplasmic reticulum lipid droplet junctions and is important for droplet morphology. Proc. Natl. Acad. Sci. USA 104, 20890-20895.

[33] Magre, J. et al. (2001) Identification of the gene altered in Berardinelli-Seip congenital lipodystrophy on chromosome 11q13. Nat. Genet. 28, 365-370.

[34] Chen, W., Yechoor, V.K., Chang, B.H., Li, M.V., March, K.L. and Chan, L. (2009) The human lipodystrophy gene product Berardinelli-Seip congenital lipodystrophy 2/seipin plays a key role in adipocyte differentiation. Endocrinology 150, 4552-4561.

[35] Boutet, E. et al. (2009) Seipin deficiency alters fatty acid Delta9 desaturation and lipid droplet formation in Berardinelli-Seip congenital lipodystrophy. Biochimie 91, 796-803.

[36] Lingwood, D. and Simons, K. (2010) Lipid rafts as a membrane-organizing principle. Science 327, 46-50.

[37] Bartz, R. et al. (2007) Lipidomics reveals that adiposomes store ether lipids and mediate phospholipid traffic. J. Lipid Res. 48, 837-847.

[38] Tauchi-Sato, K., Ozeki, S., Houjou, T., Taguchi, R. and Fujimoto, T. (2002) The surface of lipid droplets is a phospholipid monolayer with a unique fatty acid composition. J. Biol. Chem. 277, 44507-44512.

[39] Ivanova, P.T., Milne, S.B., Myers, D.S. and Brown, H.A. (2009) Lipidomics: a mass spectrometry based systems level analysis of cellular lipids. Curr. Opin. Chem. Biol. 13, 526-531.

[40] Almahbobi, G., Williams, L.J., Han, X.G. and Hall, P.F. (1993) Binding of lipid droplets and mitochondria to intermediate filaments in rat Leydig cells. J. Reprod. Fertil. 98, 209-217.

[41] Stone, S.J., Levin, M.C., Zhou, P., Han, J., Walther, T.C. and Farese Jr., R.V. (2009) The endoplasmic reticulum enzyme DGAT2 is found in mitochondriaassociated membranes and has a mitochondrial targeting signal that promotes its association with mitochondria. J. Biol. Chem. 284, 53525361.

[42] Binns, D. et al. (2006) An intimate collaboration between peroxisomes and lipid bodies. J. Cell Biol. 173, 719-731.

[43] Beller, M., Sztalryd, C., Southall, N., Bell, M., Jäckle, H., Auld, D.S. and Oliver, B. (2008) COPI complex is a regulator of lipid homeostasis. PLoS Biol. 6, e292.

[44] Guo, Y. et al. (2008) Functional genomic screen reveals genes involved in lipiddroplet formation and utilization. Nature 453, 657-661.

[45] Wan, H.C., Melo, R.C., Jin, Z., Dvorak, A.M. and Weller, P.F. (2007) Roles and origins of leukocyte lipid bodies: proteomic and ultrastructural studies. FASEB J. 21, 167-178.

[46] Dvorak, A.M., Morgan, E.S. and Weller, P.F. (2003) RNA is closely associated with human mast cell lipid bodies. Histol. Histopathol. 18, 943-968.

[47] Targett-Adams, P., Boulant, S. and McLauchlan, J. (2008) Visualization of double-stranded RNA in cells supporting hepatitis C virus RNA replication. J. Virol. 82, 2182-2195.

[48] Holthuis, J.C. and Levine, T.P. (2005) Lipid traffic: floppy drives and a superhighway. Nat. Rev. Mol. Cell Biol. 6, 209-220.

[49] Murphy, S., Martin, S. and Parton, R.G. (2009) Lipid droplet-organelle interactions; sharing the fats. Biochim. Biophys. Acta 1791, 441-447.

[50] Zehmer, J.K., Huang, Y., Peng, G., Pu, J., Anderson, R.G. and Liu, P. (2009) A role for lipid droplets in inter-membrane lipid traffic. Proteomics 9, 914-921.

[51] Welte, M.A. (2009) Fat on the move: intracellular motion of lipid droplets. Biochem. Soc. Trans. 37, 991-996.

[52] Liu, P., Bartz, R., Zehmer, J.K., Ying, Y.S., Zhu, M., Serrero, G. and Anderson, R.G. (2007) Rab-regulated interaction of early endosomes with lipid droplets. Biochim. Biophys. Acta 1773, 784-793.

[53] Takeda, Y. and Nakano, A. (2008) In vitro formation of a novel type of membrane vesicles containing Dpm1p: putative transport vesicles for lipid droplets in budding yeast. J. Biochem. 143, 803-811.

[54] Hommel, A. et al. (2010) The ARF-like GTPase ARFRP1 is essential for lipid droplet growth and is involved in the regulation of lipolysis. Mol. Cell. Biol. 30, 1231-1242. 
[55] Soni, K.G., Mardones, G.A., Sougrat, R., Smirnova, E., Jackson, C.L. and Bonifacino, J.S. (2009) Coatomer-dependent protein delivery to lipid droplets. J. Cell Sci. 122, 1834-1841.

[56] Beck, R., Rawet, M., Wieland, F.T. and Cassel, D. (2009) The COPI system: molecular mechanisms and function. FEBS Lett. 583, 2701-2709.

[57] Lee, M.C., Miller, E.A., Goldberg, J., Orci, L. and Schekman, R. (2004) Bidirectional protein transport between the ER and Golgi. Annu. Rev. Cell Dev. Biol. 20, 87-123.

[58] Nakamura, N., Akashi, T., Taneda, T., Kogo, H., Kikuchi, A. and Fujimoto, T. (2004) ADRP is dissociated from lipid droplets by ARF1-dependent mechanism. Biochem. Biophys. Res. Commun. 322, 957-965.

[59] Lay, D., Gorgas, K. and Just, W.W. (2006) Peroxisome biogenesis: where Arf and coatomer might be involved. Biochim. Biophys. Acta 1763, 1678-1687.

[60] Khaliullina, H., Panakova, D., Eugster, C., Riedel, F., Carvalho, M. and Eaton, S. (2009) Patched regulates Smoothened trafficking using lipoprotein-derived lipids. Development 136, 4111-4121.

[61] Grönke, S., Mildner, A., Fellert, S., Tennagels, N., Petry, S., Müller, G., Jäckle, H. and Kühnlein, R.P. (2005) Brummer lipase is an evolutionary conserved fat storage regulator in Drosophila. Cell Metab. 1, 323-330.
[62] Zimmermann, R. et al. (2004) Fat mobilization in adipose tissue is promoted by adipose triglyceride lipase. Science $306,1383-1386$.

[63] Haemmerle, G. et al. (2006) Defective lipolysis and altered energy metabolism in mice lacking adipose triglyceride lipase. Science 312, 734-737.

[64] Jägerström, S., Polesie, S., Wickström, Y, Johansson, B.R. Schröder, H.D. Hojlund, K. and Boström, P. (2009) Lipid droplets interact with mitochondria using SNAP23. Cell Biol. Int. 33, 934-940.

[65] Spandl, J., White, D.J., Peychl, J. and Thiele, C. (2009) Live cell multicolor imaging of lipid droplets with a new dye, LD540. Traffic 10, 1579-1584.

[66] Lee, B., Zhu, J., Wolins, N.E., Cheng, J.X. and Buhman, K.K. (2009) Differential association of adipophilin and TIP47 proteins with cytoplasmic lipid droplets in mouse enterocytes during dietary fat absorption. Biochim. Biophys. Acta $1791,1173-1180$

[67] Rinia, H.A., Burger, K.N., Bonn, M. and Müller, M. (2008) Quantitative label-free imaging of lipid composition and packing of individual cellular lipid droplets using multiplex CARS microscopy. Biophys. J. 95, 4908-4914.

[68] Yla-Anttila, P., Vihinen, H., Jokitalo, E. and Eskelinen, E.L. (2009) 3D tomography reveals connections between the phagophore and endoplasmic reticulum. Autophagy 5, 1180-1185. 